\title{
Specialized Program of Research Excellence
}

National Cancer Institute

\section{Source}

National Cancer Institute. Specialized Program of Research Excellence. NCI Thesaurus.

Code C19460.

The Specialized Programs of Research Excellence (SPOREs) established by NCI to promote interdisciplinary research and to speed the bi-directional exchange between basic and clinical science to move basic research finding from the laboratory to applied settings involving patients and populations. The goal of the SPORE program is to bring to clinical care settings novel ideas that have the potential to reduce cancer incidence and mortality, improve survival, and to improve the quality of life. SPOREs are funded through specialized center grants (P50s). 